\title{
Methods
}

The field context of all samples was studied during field campaigns to the Ries impact structure in 2000, 2001, 2005, 2009, and 2010. In addition to petrographic observations, all samples were studied at hand specimen scale. Thin sections $(30 \mu \mathrm{m})$ were prepared from each of the 119 collected samples (Table S1, Fig. S1). Petrography was completed with a Nikon Eclipse LV100POL petrographic light microscope equipped with a Nikon DS-Ri1 12 megapixel camera. Extended-depth of focus images (EDF) were obtained using plane-polarized transmission microscopy by aligning multiple images in the $\mathrm{z}$ plane using Nikon Elements software. On average $25-35$ images were collected at $\sim 0.4 \mu \mathrm{m} z$ spacing and merged to create a single EDF image. Reflected light was used to target areas for SEM analysis by identifying regions where tubules intersected the thin section surface. Eight Polished petrographic thin sections (RI 00 056, RI 00_006, RI_01_006, RI_09_006, RI_09_086, RI_10_13 5m, RI_10_009A1, RI_10_006) were coated with amorphous Os and sample RI_10_006 was also prepared for secondary electron (SE) imaging. SE stubs were prepared by dipping $\mathrm{C}$-tape coated Ti scanning electron microscopy (SEM) stubs into crushed ( 1 mm grain size) glass clasts. Sections were analyzed with back-scattered (BSE) and secondary electron imaging and energy dispersive X-ray (EDX) spectroscopy carried out with a Leo 1540 FIB/SEM CrossBeam field emission SEM equipped with an Oxford Instruments INCA EDX system sensitive to $\sim 0.5 \mathrm{wt}$. \% or less for all elements from $\mathrm{C}-\mathrm{U}$. Qualitative relative elemental ratios are based on normalized spectral intensities from linescans produced from EDX elemental maps. Fourier Transform Infra-Red (FTIR) spectroscopy was carried out on both tubulerich and tubule-free areas (as indicated in Fig. S6) of sample RI_00_056 using a Bruker IFS55 FTIR with a Baseline TM Horizontal Attenuated Total Reflection (ATR) attachment equipped with a germanium crystal, under an IRScope II microscope.

\begin{tabular}{lccc}
\multicolumn{3}{c}{ TABLE DR1. SAMPLE LOCATIONS } \\
\hline \hline Sample \# & Locality & Easting & Northing \\
\hline $00-001$ & Otting & 631628 & 5415240 \\
$00-002$ & Otting & 631628 & 5415240 \\
$00-005$ & Otting & 631628 & 5415240 \\
$00-006$ & Otting & 631628 & 5415240 \\
$00-007$ & Otting & 631628 & 5415240 \\
$00-009$ & Otting & 631628 & 5415240 \\
$00-012$ & Aumühle & 619650 & 5425083 \\
$00-013$ & Aumühle & 619650 & 5425083 \\
$00-014$ & Aumühle & 619650 & 5425083
\end{tabular}




\begin{tabular}{|c|c|c|c|}
\hline 00-018 & Aumühle & 619650 & 5425083 \\
\hline $00-024$ & Aumühle & 619650 & 5425083 \\
\hline $00-025$ & Aumühle & 619650 & 5425083 \\
\hline $00-028$ & Zipplingen & 603707 & 5420198 \\
\hline $00-029$ & Zipplingen & 603707 & 5420198 \\
\hline $00-030$ & Zipplingen & 603707 & 5420198 \\
\hline 00-031 & Zipplingen & 603707 & 5420198 \\
\hline 00-032 & Zipplingen & 603707 & 5420198 \\
\hline $00-048$ & Altenburg & 725204 & 5411352 \\
\hline 00-049 & Seelbronn & 608087 & 5399128 \\
\hline $00-050$ & Seelbronn & 608087 & 5399128 \\
\hline 00-051 & Seelbronn & 608087 & 5399128 \\
\hline 00-052 & Seelbronn & 608087 & 5399128 \\
\hline $00-055$ & Amerdingen & 609449 & 5397705 \\
\hline 00-056 & Amerdingen & 609449 & 5397705 \\
\hline $00-057$ & Amerdingen & 609449 & 5397705 \\
\hline 00-059 & Sternbach & 610930 & 5399375 \\
\hline $00-060$ & Sternbach & 610930 & 5399375 \\
\hline 00-061 & Mauren & 623336 & 5402794 \\
\hline 01-003 & Aumühle & 619650 & 5425083 \\
\hline 01-004 & Aumühle & 619650 & 5425083 \\
\hline 01-006 & Aumühle & 619650 & 5425083 \\
\hline 01-007 & Aumühle & 619650 & 5425083 \\
\hline 01-008 & Aumühle & 619650 & 5425083 \\
\hline 01-009 & Aumühle & 619650 & 5425083 \\
\hline $01-010$ & Aumühle & 619650 & 5425083 \\
\hline 01-024 & Zipplingen & 603707 & 5420198 \\
\hline 01-025 & Zipplingen & 603707 & 5420198 \\
\hline 01-027 & Aufhausen & 597832 & 5412330 \\
\hline $01-028$ & Aufhausen & 597832 & 5412330 \\
\hline 01-029 & Aufhausen & 597832 & 5412330 \\
\hline 01-030 & Anhausen & 608615 & 5404454 \\
\hline 01-031 & Anhausen & 608615 & 5404454 \\
\hline 05-007 & Otting & 631628 & 5415240 \\
\hline $05-008$ & Otting & 631628 & 5415240 \\
\hline $05-013$ & Aumühle & 619650 & 5425083 \\
\hline 05-015 & Aumühle & 619650 & 5425083 \\
\hline 05-016 & Aumühle & 619650 & 5425083 \\
\hline 05-018 & Aumühle & 619650 & 5425083 \\
\hline $05-020$ & Aumühle & 619650 & 5425083 \\
\hline 05-021 & Aumühle & 619650 & 5425083 \\
\hline $05-022$ & Aumühle & 619650 & 5425083 \\
\hline $05-025$ & Sternbach & 610930 & 5399375 \\
\hline
\end{tabular}




\begin{tabular}{|c|c|c|c|}
\hline 05-032 & Wengenhausen & 607663 & 5418266 \\
\hline 05-033 & Unterwilfingen & 606178 & 5419091 \\
\hline 09-004 & Unterwilfingen & 605971 & 5419047 \\
\hline 09-005 & Zipplingen & 603207 & 5420223 \\
\hline 09-006 & Seelbron & 608073 & 5399066 \\
\hline 09-007 & Seelbron & 608073 & 5399066 \\
\hline 09-008a & Seelbron & 608073 & 5399066 \\
\hline 09-008b & Seelbron & 608073 & 5399066 \\
\hline 09-009 & Seelbron & 608073 & 5399066 \\
\hline 09-010a & Seelbron & 608073 & 5399066 \\
\hline 09-010b & Seelbron & 608073 & 5399066 \\
\hline 09-011 & Seelbron & 608073 & 5399066 \\
\hline 09-012 & Seelbron & 608073 & 5399066 \\
\hline $09-013$ & Seelbron & 608073 & 5399066 \\
\hline 09-014 & Altenburg & 605060 & 5407674 \\
\hline 09-015 & Altenburg & 605060 & 5407674 \\
\hline 09-020 & Sternbach & 610930 & 5399375 \\
\hline $09-021$ & Sternbach & 610930 & 5399375 \\
\hline 09-022 & Sternbach & 610930 & 5399375 \\
\hline 09-023 & Sternbach & 610930 & 5399375 \\
\hline 09-024 & Sternbach & 610930 & 5399375 \\
\hline 09-036 & Altenburg & 605060 & 5407674 \\
\hline $09-037$ & Altenburg & 605060 & 5407674 \\
\hline $09-038$ & Altenburg & 605060 & 5407674 \\
\hline 09-039 & Aumühle & 619171 & 5425521 \\
\hline $09-040$ & Aumühle & 619171 & 5425521 \\
\hline $09-043$ & Aumühle & 619171 & 5425521 \\
\hline 09-046 & Aumühle & 618670 & 5425560 \\
\hline 09-047 & Aumühle & 618670 & 5425560 \\
\hline 09-049 & Aumühle & 618670 & 5425560 \\
\hline 09-051 & Aumühle & 618670 & 5425560 \\
\hline 09-052 & Otting & 631319 & 5415357 \\
\hline $10-0050 \mathrm{~cm}$ & Aumühle & 618670 & 5425560 \\
\hline $10-00550 \mathrm{~cm}$ & Aumühle & 618670 & 5425560 \\
\hline $10-00560 \mathrm{~cm}$ & Aumühle & 618670 & 5425560 \\
\hline $10-00570 \mathrm{~cm}$ & Aumühle & 618670 & 5425560 \\
\hline $10-0051 \mathrm{~m}$ & Aumühle & 618670 & 5425560 \\
\hline $10-0051.5 \mathrm{~m}$ & Aumühle & 618670 & 5425560 \\
\hline $10-0052 m$ & Aumühle & 618670 & 5425560 \\
\hline $10-0052.5 \mathrm{~m}$ & Aumühle & 618670 & 5425560 \\
\hline $10-0053 m$ & Aumühle & 618670 & 5425560 \\
\hline $10-0053.5 \mathrm{~m}$ & Aumühle & 618670 & 5425560 \\
\hline $10-0054 m$ & Aumühle & 618670 & 5425560 \\
\hline
\end{tabular}




\begin{tabular}{|c|c|c|c|}
\hline $10-0054.5 \mathrm{~m}$ & Aumühle & 618670 & 5425560 \\
\hline $10-0055 m$ & Aumühle & 618670 & 5425560 \\
\hline $10-009$ & Amerdingen & 609449 & 5397705 \\
\hline 10-012 0m & Aumühle & 619171 & 5425521 \\
\hline 10-012 1m & Aumühle & 619171 & 5425521 \\
\hline $10-0122 m$ & Aumühle & 619171 & 5425521 \\
\hline $10-0123 m$ & Aumühle & 619171 & 5425521 \\
\hline $10-0124 m$ & Aumühle & 619171 & 5425521 \\
\hline $10-0125 \mathrm{~m}$ & Aumühle & 619171 & 5425521 \\
\hline $10-0126 m$ & Aumühle & 619171 & 5425521 \\
\hline $10-0127 m$ & Aumühle & 619171 & 5425521 \\
\hline $10-0128 m$ & Aumühle & 619171 & 5425521 \\
\hline 10-013 0m & Aumühle & 619171 & 5425521 \\
\hline 10-013 1m & Aumühle & 619171 & 5425521 \\
\hline $10-0132 m$ & Aumühle & 619171 & 5425521 \\
\hline 10-013 3m & Aumühle & 619171 & 5425521 \\
\hline $10-0134 m$ & Aumühle & 619171 & 5425521 \\
\hline $10-0135 \mathrm{~m}$ & Aumühle & 619171 & 5425521 \\
\hline $10-0136 m$ & Aumühle & 619171 & 5425521 \\
\hline $10-0200 \mathrm{~cm}$ & Aumühle & 619171 & 5425521 \\
\hline $10-02050 \mathrm{~cm}$ & Aumühle & 619171 & 5425521 \\
\hline $10-0201 \mathrm{~m}$ & Aumühle & 619171 & 5425521 \\
\hline $10-0201.5 \mathrm{~m}$ & Aumühle & 619171 & 5425521 \\
\hline $10-0202 m$ & Aumühle & 619171 & 5425521 \\
\hline $10-021$ & Aumühle & 619171 & 5425521 \\
\hline
\end{tabular}




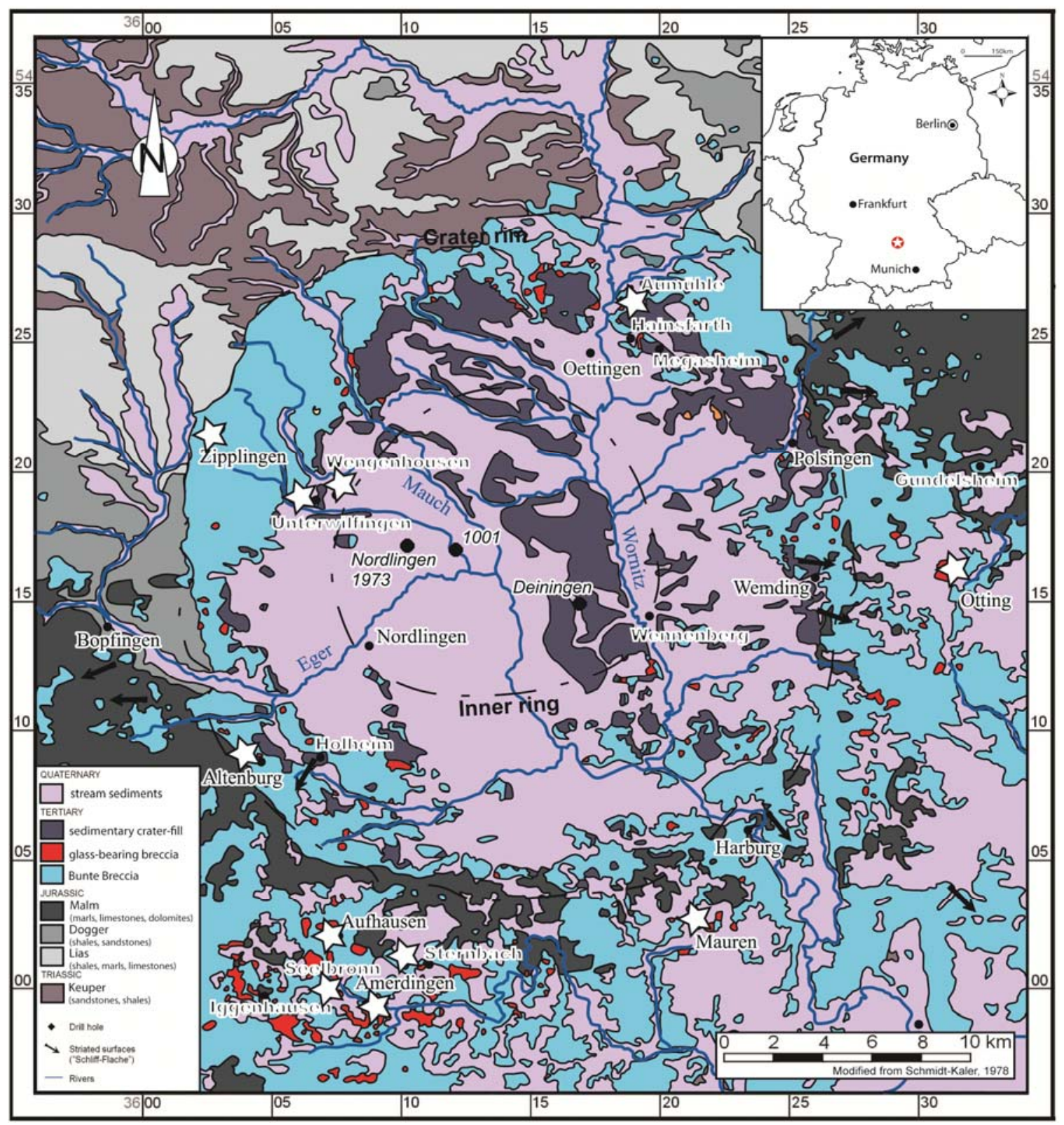

Figure DR1: Simplified geologic map of the Ries impact structure, with sample locations. Modified from Osinski (2003). The inner dotted line delineates the crystalline inner ring of uplifted basement that surrounds the $\sim 12 \mathrm{~km}$ inner basin. The outer dotted line marks the $\sim 24 \mathrm{~km}$ diameter crater rim. Samples were obtained from the indicated locations (white stars) representing the spatial distribution of the impactite outcrops. 


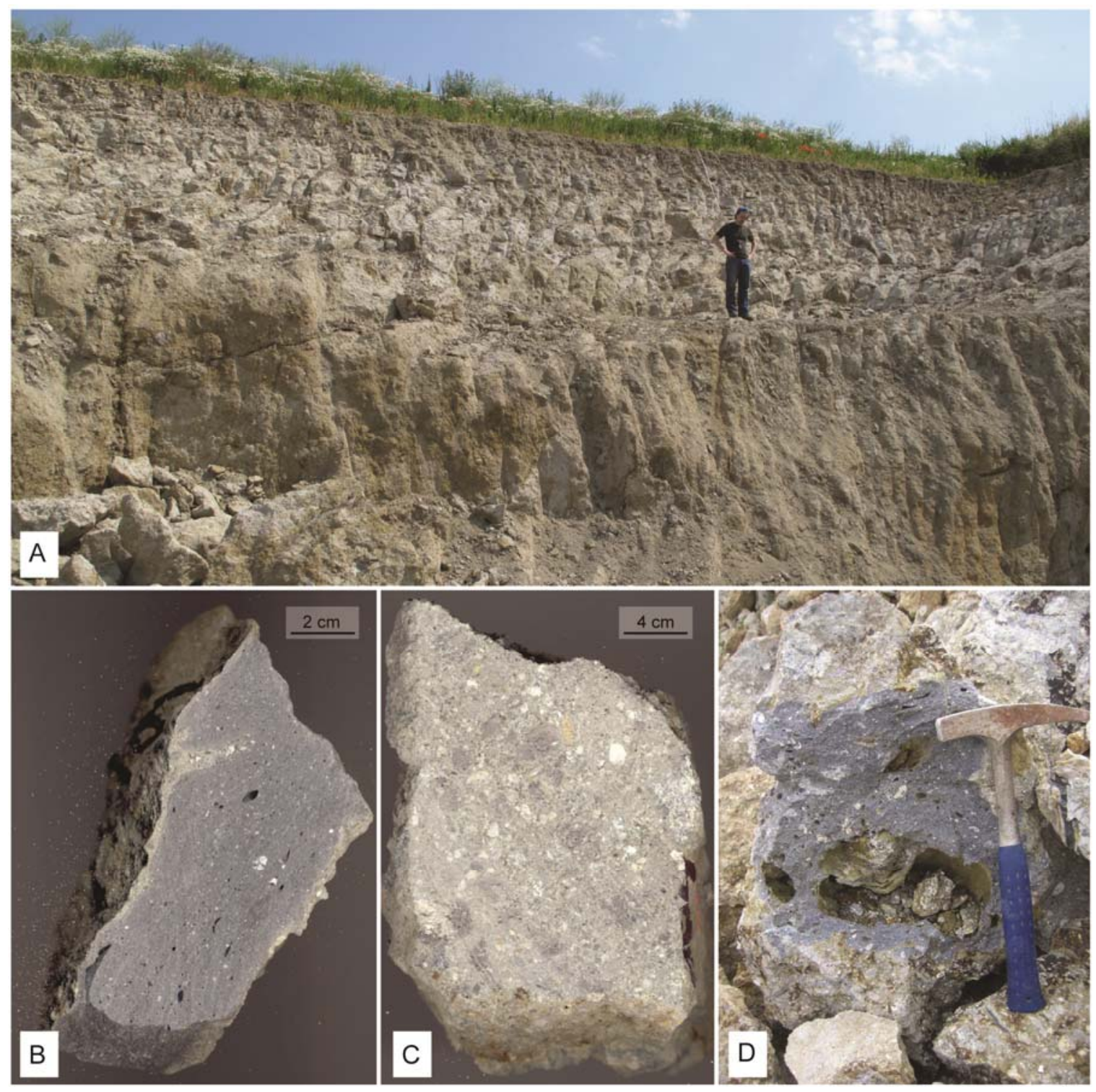

Figure DR2: Field and hand sample photographs of the Ries glass-bearing breccia. A North wall of Aumühle quarry exposing over $6 \mathrm{~m}$ of glass-bearing breccia (surficial suevite). B Relatively un-weathered impact glass from Otting (RI_00_006). C weathered sample of glass bearing breccia containing lithic and altered glass clasts from Amerdingen (RI_10_009). D. Large vesicular glass clast from Seelbronn quarry. 


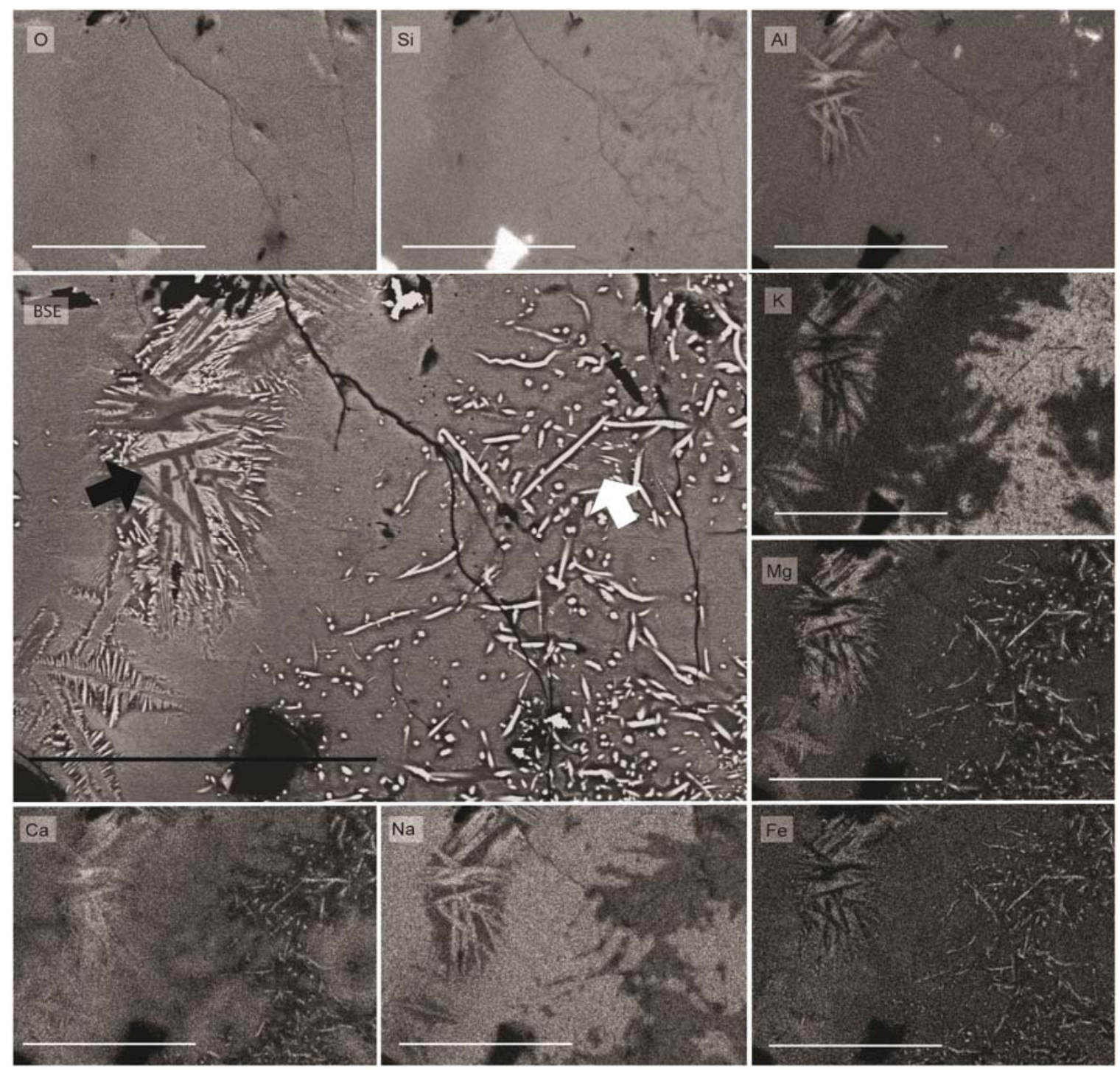

Figure DR3: EDX mapping of lack of variation in host glass chemistry between areas hosting crystallites (left on BSE image: black arrow indicating dark plagioclase laths) and tubular features (right on BSE image: white arrow). Scale bars $50 \mu \mathrm{m}$. Sample RI_00_006. 

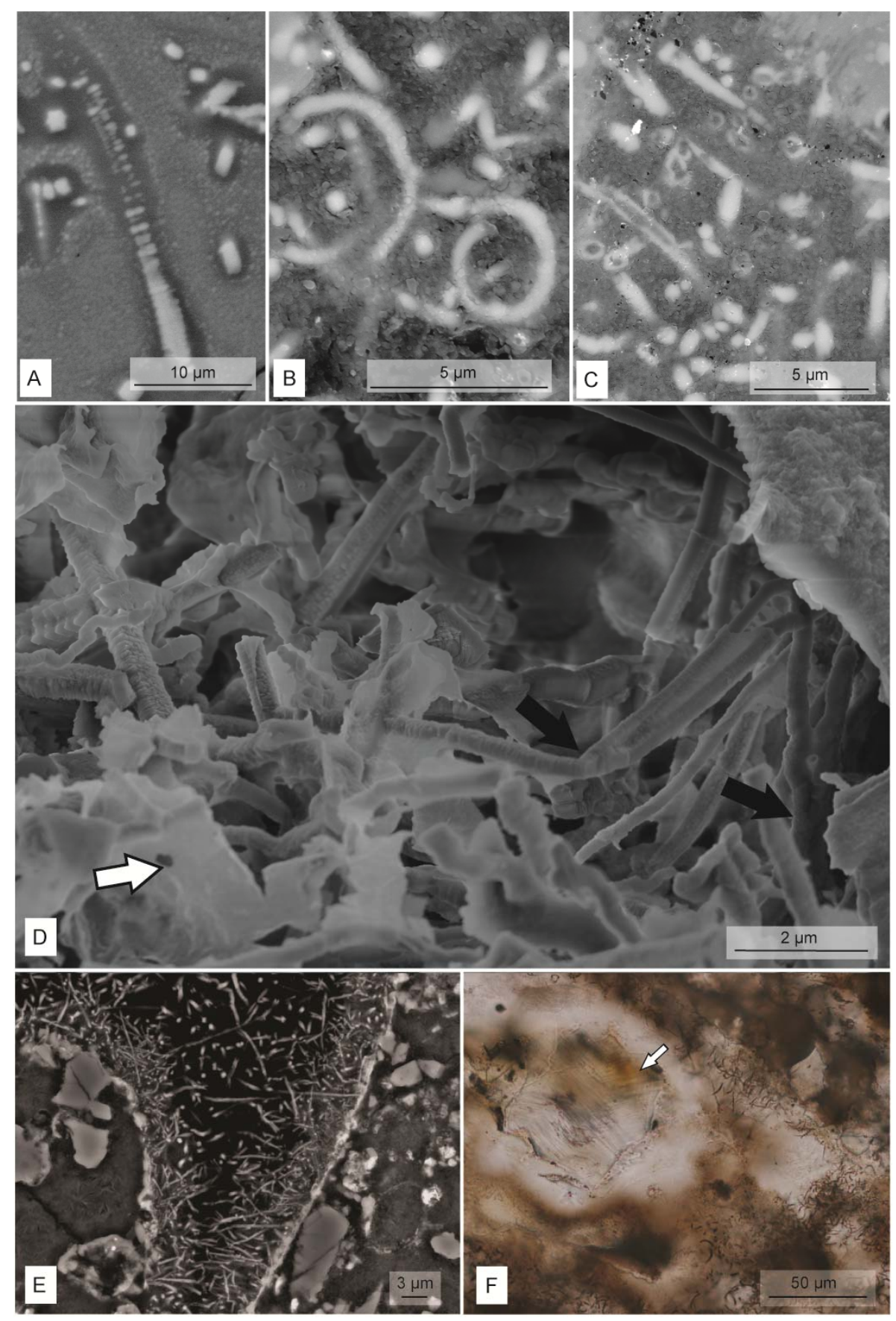

Figure DR4: Photomicrographs of tubules in Ries glasses. A BSE image of tubule feature with ornamented margins (RI_00_056). B BSE image of solid, smooth-walled tubules (RI_00_056). C BSE image of hollow, smooth-walled tubules (RI_00_056). D SE imaging mode illustration film-like material reminiscent of EPS associated with the tubules (white arrow). Also note the branching (black arrow) of tubules where the daughter tubules are the same width as the parent tubules (RI_10_006). E BSE imaging mode illustrating the association of tubules with the margin of the glass clast (RI_10_009A1). F Note the absence of tubules in Si-rich areas of glass surrounding a partially resorbed shocked quartz clast displaying planar deformation features (RI_10_013 $4 \mathrm{~m}$ ). 


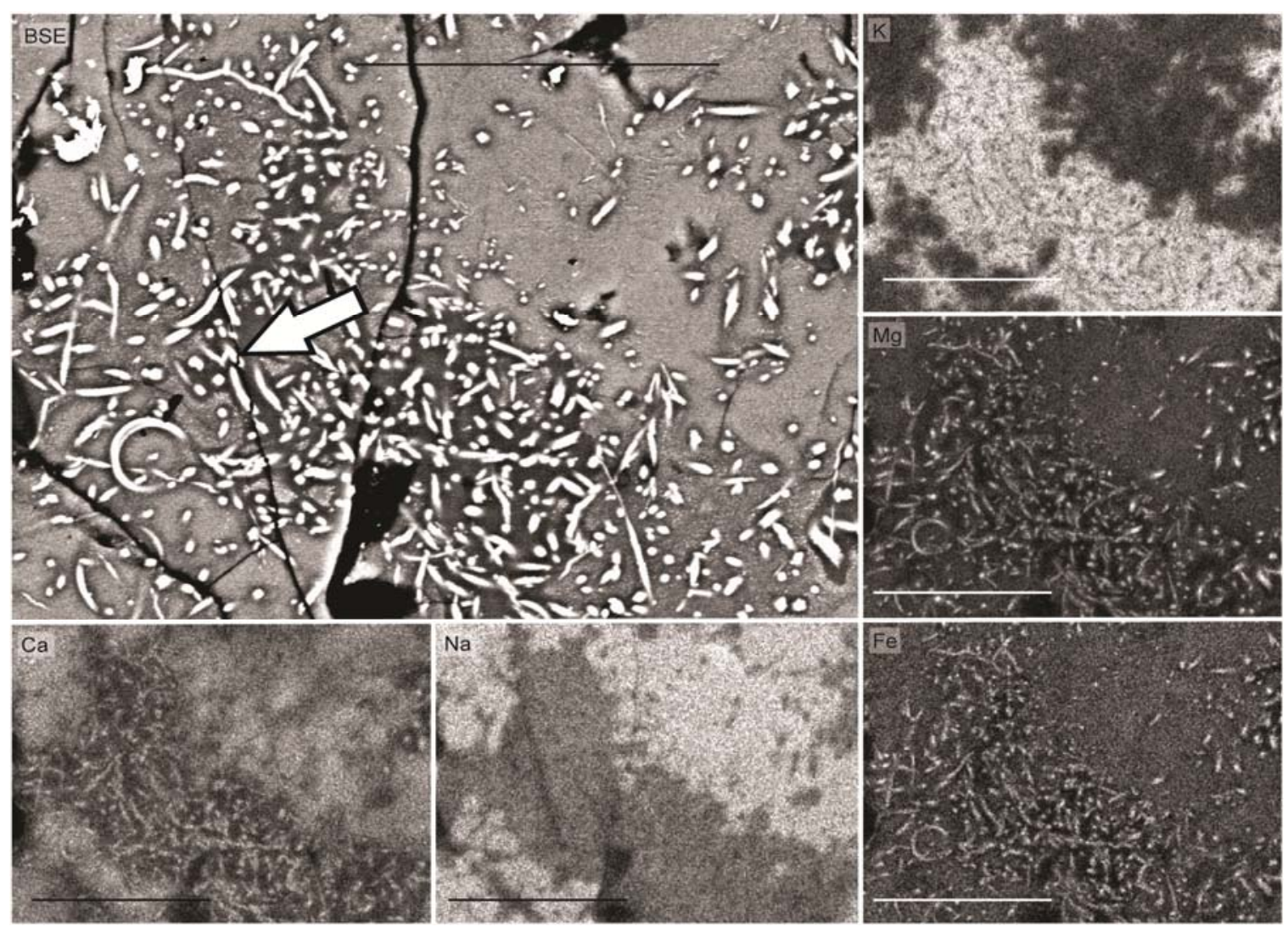

Figure DR5: EDX mapping illustrating matrix composition surrounding tubular features. Tubules surrounded by zone depleted in $\mathrm{Ca}$, $\mathrm{Na}$, $\mathrm{Fe}$ and $\mathrm{Mg}$ while enriched in $\mathrm{K}$. Tubules are enriched in $\mathrm{Ca}, \mathrm{Fe}$ and $\mathrm{Mg}$. Scale bars $30 \mu \mathrm{m}$. Sample RI_09_006 


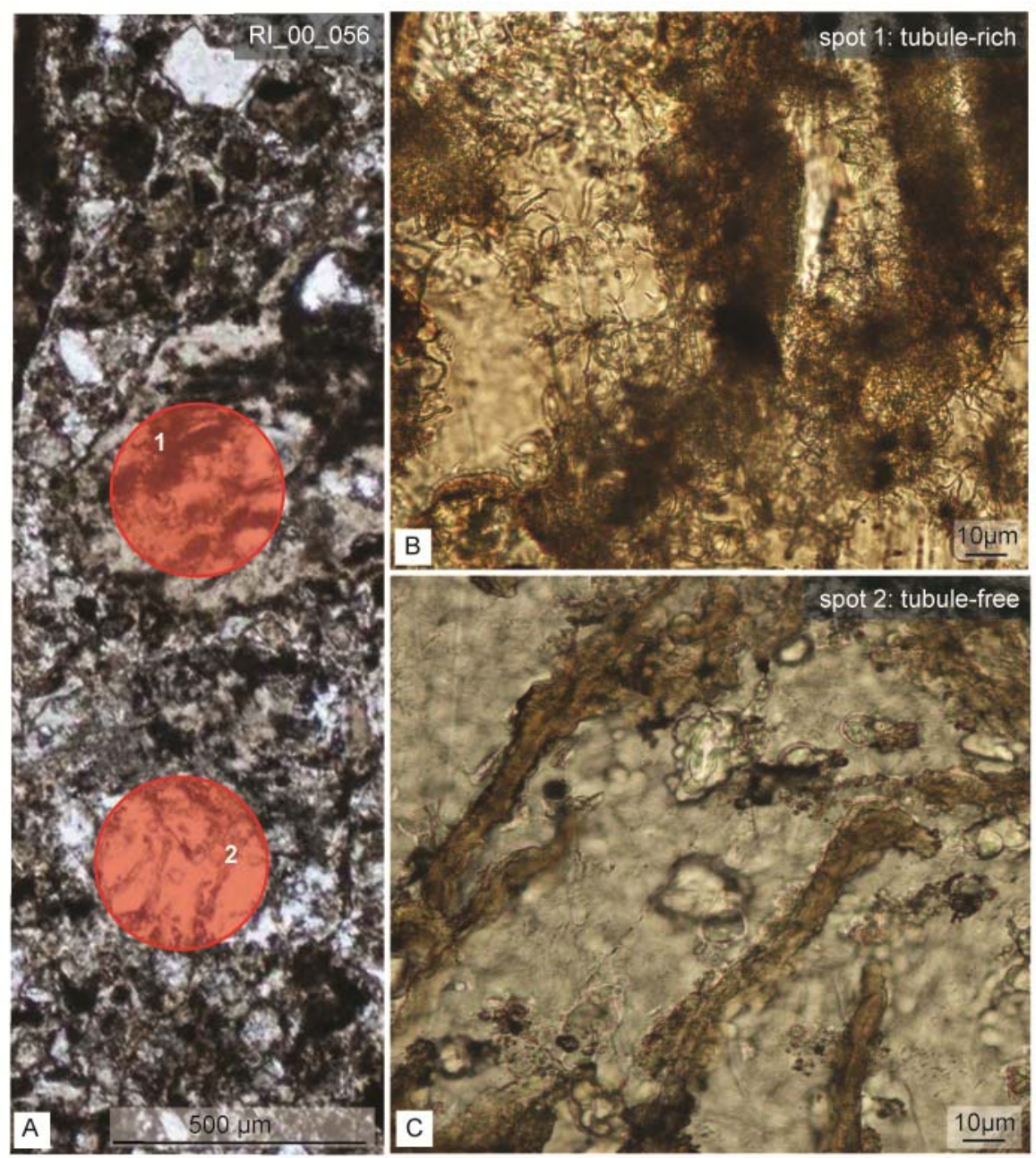

Figure DR6: Transmitted light images of the tubule-rich area (spot 1) and tubule-free area (spot 2) corresponding to FTIR absorbance spectra. A photomicrograph of a glassbearing breccia indicating areas of FTIR analyses (red circles). B Spot 1, a tubule-rich area. C Spot 2, a tubule-free area. Sample RI_00_056.

\section{Supplementary Reference}

Schmidt-Kaler H. 1978. Geological setting and history, in Chao E. C. T., Hüttner R., and Schmidt-Kaler K., eds., Principle exposures of the Ries meteorite crater in southern Germany: Munich, Bayerisches Geologisches Landesamt, p. 8-11. 